\title{
Communicating about Communication: Intercultural competence as a factor in the success of interdisciplinary collaboration
}

\author{
Helen Fraser \\ University of New England \\ hfraser@une.edu.au
}

\author{
Andrea C. Schalley \\ Griffith University \\ a.schalley@griffith.edu.au
}

\author{
Consulting author: Andrea C. Schalley \\ School of Languages and Linguistics \\ Griffith University \\ Nathan, QLD 4111
}

Note: The order of authors is alphabetical. Both authors contributed equally to the final product. 


\title{
Communicating about Communication: \\ Intercultural competence as a factor in the success of interdisciplinary collaboration
}

\begin{abstract}
Many disciplines describe themselves as studying 'communication'. However observation of interdisciplinary discussion suggests that 'communication' may be conceptualized in different ways by different disciplines. This paper aims to promote mutual understanding among disciplines, not by proposing a universally valid definition of communication to which all disciplines should subscribe, but by, first, offering a set of questions that can be used to help disciplinary groups communicate their own views on communication to colleagues from other disciplines, and then creating a (preliminary) typology to map out the range of possible positions that can be taken in relation to those questions. Noting that academic disciplines have distinct cultures, the paper presents some concepts of intercultural communication as understood in applied linguistics that may be useful in facilitating interdisciplinary communication about communication.
\end{abstract}

\section{Keywords}

communication, interdisciplinary collaboration, terminology, intercultural competence

"Collaboration - across disciplines, sectors and geographic boundaries - is central to the creation and success of an innovative country." Senator Kim Carr, Minister for Innovation, Industry, Science and Research, May 2008

\section{Introduction}

Interdisciplinary collaboration can be spectacularly successful. The development of cognitive science is just one notable example of its potential (Miller 2003). For this reason, projects featuring interdisciplinary collaboration have come to be highly prized by government and other funding agencies. The problem is that successful interdisciplinary projects tend to arise naturally through cooperation born of shared interests and common aims. When externally mandated, interdisciplinary collaboration can be surprisingly difficult to achieve (Lele \& Norgaard 2005). Much effort has gone into studying the difficulties involved, to the extent we may be witnessing the birth of a new discipline called Interdisciplinary Studies (Augsburg 2006, Klein 1990, Moran 2001). Such research has identified a number of barriers to interdisciplinary collaboration - from the physical arrangement of buildings on campuses, to deep-seated mutual suspicion between advocates of quantitative versus qualitative methodologies (Todd et al. 2001) - and recommended strategies for overcoming them (Derry et al. 2005, Lattuca 2001, Weingart \& Stehr 2000). 
One barrier which, though undoubtedly recognized as important (Lattuca 2001), has received surprisingly little attention, is the simple fact that the same words can mean different things in different disciplines. While discipline-specific technical terms may be readily identified, and explained to colleagues from other disciplines, some very basic terminology appears to be common to a wide range of different disciplines, giving a sense that no explanation is needed. However, similarity in the form of these terms can mask widely different conceptualizations, which, if not uncovered, can cause misunderstanding and frustration.

One such basic term is 'communication'. Even in everyday usage, the familiarity of this word masks considerable complexity of meaning. With etymological roots in medieval notions of 'making public (or "common"), as opposed to private' (Macquarie Dictionary), over the centuries it has developed a richly interlocking tapestry of meanings and cultural connotations (Bennett et al. 2005). During these centuries, it has also been the subject of academic research in a range of disciplines. In the twentiethcentury, a particular definition of communication as 'information transfer' was brought to prominence by the technical discipline of telephony and communications engineering (Shannon \& Weaver 1949), and for a time served as a unifying theme for the interdisciplinary study of communication. However in recent times, this unity seems to have been lost, with some disciplines extending the definition in novel ways, while others put forward serious criticisms of the idea of communication as a simple transfer of information (Chandler 1994, Liddicoat, this vol., Haugh, this vol).

The fact that communication can be conceptualized in different ways by different disciplines was highlighted by the discussion accompanying the formation of the Human Communication Science Network (HCSNet) in 2004-5. HCSNet is one of several Australian Research Council (ARC) Research Networks, "designed to encourage collaborative approaches to research in inter-disciplinary settings" (ARC website http://www.arc.gov.au/ncgp/networks/networks_default.htm). Its formation brought together a large number of academics studying communication in a wide range of disciplines, from Natural Language Processing, to Speech Pathology, to Dance. Preliminary meetings of participants suggested, however, that in some cases, different people had different ideas of what communication actually is. The workshop 'Conceptualising Communication', co-hosted by HCSNet and the University of New England's Language and Cognition Research Centre (LCRC) was held in December 2005 at UNE, Armidale NSW, to explore this topic.

Participants were enthusiastic in their feedback that the workshop had opened their eyes to previously unnoticed differences regarding what 'communication' means in different disciplines, and unanimous in their recognition of a need for improved mutual understanding of the usage of this key term. However there was strong resistance to the idea of creating a new, all-encompassing definition of 'communication' to which each discipline would have to sign up. While members of each discipline were interested to explore the interpretations that others put on this - and other - key terms, ultimately they wanted to be able to return to their own interpretations. Nor was there great enthusiasm for 
'tutorials' from one discipline for another, with their implication of a teacher-student relationship. As one participant (Prof Michael Wagner, University of Canberra) put it, what we need is a series of 'Lonely Planet' guides for the disciplines, in which each provides an overview and background suitable for tourists from other disciplines. Several of the papers in the current volume can perhaps be seen as examples of this new genre of writing.

The present paper takes this idea one step further, by recognising that the interdisciplinary study of communication requires not just studying communication, but also doing communication - it is communication about communication. The idea that academic disciplines have their own cultures (Becher \& Trowler 2001) raises an analogy with intercultural communication. In intercultural communication, it is commonplace for the same word, or its translation equivalent, to refer to widely differing concepts. Thus people from different cultures can have very different ideas of what it means to 'show respect', 'be polite', or 'love someone' (Wierzbicka 1997). Intercultural communication can be facilitated by providing a space for interactants to discuss and explore what such terms mean to them (Byrne 1996, FitzGerald 2003). This is one step in development of intercultural competence (Byram et al. 2001) - a skill that we will argue later is a key to successful interdisciplinary collaboration.

This paper, then, has two main aims. The first is to encourage and facilitate interdisciplinary discussion about the nature of communication, with a view to improving mutual understanding, and easing the process of collaboration across disciplinary boundaries. Such discussion may be particularly important for graduate students, enabling them to 'grow up bilingual' in more than one discipline rather than always remaining 'second language speakers'. This aim is approached through providing a series of discussion questions intended to help disciplinary groups make their understanding of 'communication' explicit to interdisciplinary colleagues, and throw up points of disagreement to be worked through. Essentially the intention is to capture, recreate and extend some of the flavour of the productive discussion at ConCom05 (Schalley et al. 2005).

The second aim is to extend the 'Lonely Planet' idea by providing a map or typology of some of the different positions that can be taken with regard to the nature and study of communication. This aim is approached by attempting to abstract dimensions of difference from the papers presented, both at Concom05 and in the present volume, with respect to their understanding of 'communication'. The purpose of the map is twofold. First it provides a broader perspective within which discussants can locate themselves in relation to other possible views and discover connections between various disciplines. Second it shows up interesting relationships between various answers to the discussion questions.

Two points are worth emphasising before we get started. First, neither of these aims is in any way directed towards pigeonholing or constraining disciplinary perspectives. The intention is entirely one of opening a space for communication about communication - a space in which views about this important keyword can be exchanged, understood and developed. Second, we make no claim to have 
canvassed all disciplines, or all views on communication within each discipline. We have based our analysis on the disciplines represented at ConCom05 and in the present volume. We are well aware that there are many other disciplines that study communication. Particularly notable omissions from ConCom05, for example, were representatives of communication/media studies (Marris \& Thornham 1996) or animal communication (Rogers \& Kaplan 1998). In short, our analysis is offered not as a final product but as a starting point.

\section{Discussion questions}

As explained above, this section suggests a set of questions intended to stimulate discussion about communication in an interdisciplinary group. The overarching question can be framed as follows:

- What does it take, in your discipline, for an event to be categorized as 'communication', and why?

Perhaps the best starting point for discussion is a brainstorming session to elicit events considered by each discipline to be

- typical examples of communication

- more peripheral examples of communication

- only metaphorically described as communication

- not communication at all.

Events to stimulate discussion can be found in any introductory text (Chandler 2002) but the following might provide a starting point:

- two people talking the same language, but one failing to understand the intended meaning of the other

- a politician using rhetoric to sway the opinion of a crowd

- a tourist using gestures and facial expressions to obtain information in a foreign land

- bees dancing to provide information about sources of pollen

- computers linked in a network

- a person typing search terms into Google

- electrical impulses traveling from the retina to the visual cortex

- a style of hair or clothing showing membership of a social group

As the examples are being categorized, the discussion group may wish not just to note differences of opinion, but to analyze which aspects of the events affect how they are categorized by different disciplines. The two sets of questions in the following sections may serve as an aid for this more detailed discussion.

\subsection{The Nature of Communication}

The first set of questions relate to the nature of communication itself. It will be seen that these questions are framed around the view of communication as transfer of a message from a sender to a receiver. This does not imply endorsement of this view as universally valid, or 'neutral'. As already 
mentioned, this view has been criticised in a number of ways - not least by papers in the present volume, which demonstrate, for example, many ways in which 'receiver' of a message is far from the passive role suggested by this term. Nevertheless, we retained the message-transfer terminology for ease of interdisciplinary comparability and facilitation of cross-disciplinary understanding. Our approach simply recognises this as the most widely-known view of communication - indeed one close to the everyday concept of communication in contemporary English, as shown by dictionary definitions like the following:

- the process by which information is exchanged between individuals through a common system of symbols, signs, or behavior; exchange of information (Merriam Webster)

- the process of transferring information from a sender to a receiver with the use of a medium (Wikipedia)

This makes it accessible to non-specialists and suitable as a starting point for discussion of communication by practitioners of a wide range of disciplines, as at ConCom05. As will be seen, the questions are intended to uncover points of disagreement with this model of communication and enable that disagreement to be expressed as clearly as possible, even to colleagues from disciplines where the message-transfer view has not (yet) been questioned.

\subsubsection{The sender}

Is it essential for a communication event to have a specific, individual sender?

If so, is the sender necessarily or typically:

- human

- animal

- machine

- part of a (biological, computational, other) system?

If not, where can messages originate from?

Must the sender have the intention of communicating?

If so, how can this intention be known/verified

- reported conscious awareness by the sender

- behaviour of the sender

If not, how is the sender of a message identified?

\subsubsection{The receiver}

Is it essential for a communication event to have a specific, individual receiver?

If so, is the receiver necessarily or typically:

- human

- animal

- machine

- part of a (biological, computational, other) system?

If not, where can messages go? 
Must the receiver understand the message?

If so, how can this understanding be known/verified

- conscious awareness by the receiver

- behaviour of the receiver

If not, how is the receiver of a message identified?

\subsubsection{The message}

Is the message necessarily or typically transferred in physical form (e.g. a letter)

If so, what physical forms can messages take?

If not, in what form are messages conveyed?

Is the message necessarily or typically 'information'?

If so, define information

If not, what is it?

- meaning

- 'content'

- information in context

- other

Is the message necessarily or typically symbolic (i.e. does it have a content independent of its physical form)?

If so, are the symbols necessarily or typically part of an underlying system such as:

- a human language

- a fixed code (identical for sender and receiver)

- a variable code (context dependent)

- another kind of semiotic system

How is that system learned or acquired by the sender/receiver?

Is the content of the message necessarily or typically an independent, determinate entity?

If so, who determines the content of the message?

- the sender

- the receiver

- sender and receiver together

- other

If not

- how does it emerge?

- is it necessarily or typically the same for sender and receiver? 


\subsection{Disciplinary values for the study of communication}

In answering questions like those in the last section, it is inevitable that some reference will be made, directly or indirectly, to the cultural values of the discipline in relation to how the academic study of communication should be undertaken. The aim of the next set of questions is to bring those cultural values into the open and make them as explicit as possible.

\subsubsection{Focus}

Is the discipline primarily concerned with communication by

- humans

- animals

- machines

- systems

- other

Does the discipline study communication as an end in itself, or does it study something else that builds on a definition of or presuppositions about communication?

Does the discipline see its definition of communication as an attempt to understand the true nature of communication, or as a convenient model of or metaphor for communication?

\subsubsection{Goals}

Is the main goal theoretical (understanding communication), practical (improving communication), or both?

Who are the main beneficiaries or addressees of the research

- academic peers

- industry partners

- practitioners

- the wider community

- other

\subsubsection{Metaphysical orientation}

Does academic research essentially discover or interpret the nature of reality?

Is human cognition (including language) essentially a formal system?

Which methods best serve to increase academic knowledge?

- quantitative

- qualitative

- formal description 
- non-formal description

What are the major criteria for good academic research?

- philosophical rigour

- a formal model

- accounting for observations

- practical outcomes or applications

- other

\section{Dimensions of difference}

The second aim of this paper, as will be recalled, is to begin development of a map or typology of the range of disciplinary positions regarding the nature of communication and approaches to its study. This is intended to help users see the range of possible positions other than their own, and understand the relationships among those positions.

This has been undertaken with respect to the (sub)disciplines represented by papers at ConCom05 namely Natural Language Processing (Dale 2005), Speech Science (Wagner 2005), Al/Information Systems (Eklund 2005), Neuropsychology (Corballis 2005), Archaeology (Davidson 2005), Music/Dance (Dean 2005; Stevens 2005), and Gesture/Sign Language (Lovell 2005; Schembri \& Johnston 2005), Intercultural Communication (Liddicoat, this vol.), Introductory Linguistics (Curnow, this vol.), Pragmatics (Haugh, this vol.), and the 'folk view' of 'communication' in contemporary English Goddard (this vol.) - supplemented by disciplines represented in papers commissioned specially for the present volume - namely Speech Pathology (Gould, this vol.), Second Language Acquisition (Eisenchlas, this vol.), Conversation Analysis (Nevile and Rendle-Short, this vol.).

By analyzing each of these works with the questions from the above section in mind, we have attempted to abstract a series of dimensions along which disciplinary views appear to vary, and created a table plotting the position taken by each of the disciplines under consideration in relation to each dimension. For this purpose we have used the views presented by the authors of the various papers as representative of their discipline. Of course we are well aware that none of the disciplines represented is monolithic. Others in the same discipline may well take a different view. Indeed the authors themselves may disagree with our characterization of how 'communication' is conceptualized in their paper. Hopefully any such disagreement would be seen as a spur to discussion.

\subsection{The nature of communication}

The discussion questions of the previous section naturally serve as a lead to the identification of such dimensions, well supplemented by the contributions to this volume and the ConCom05 presentations. Then, the simplified picture of communication as the sending of a message by a sender to a receiver via a channel, possibly deploying a system for representation of the message, facilitates the structuring of the dimensions of difference about the nature of communication. Differences can thus be expected regarding (i) the sender, (ii) the receiver, (iii) the message, and (iv) the underlying system 
used for the representation of the message. (The physical nature of the channel is disregarded in what follows, as our results show that it can generally vary without changing the definition or understanding of the nature of communication.)

One dimension which is relevant to all four above-listed aspects of communication is the core question of which 'type' (of sender, receiver, message, and underlying system) we are dealing with. In addition to this, for sender and receiver the 'criteria' for sending and receiving, respectively, are relevant, whereas for message and the underlying system the dimensions 'emergence' and 'character' surfaced. All of them will be discussed in more detail in the following sections, and they and the disciplines' positioning with respect to them are listed in Table 1.

In order to help patterns to emerge, we have plotted the disciplines on the horizontal axis of the table, and ordered the dimensions of difference on the vertical axis. The table comprises each dimension as an attribute or characteristic of - if applicable - the sender, receiver, message, or underlying system, with different values listed for each dimension. The values are (indicated by a plus), are not (indicated by a minus) or are potentially (indicated by a question mark) adopted by a discipline. For example, the attribute 'type' of the sender contains the values 'none', 'human', 'animal', 'machine', 'part of a system (biologic., computational, etc.)'. Whereas Second Language Acquisition (SLA) adopts the value 'human', Neuropsychology adopts 'part of a system (biologic., computational, etc.)' and rejects 'human'. That is, in a communication event as conceived of in SLA the sender can be human, whereas in a communication event as understood by neuropsychology it cannot be human, but is rather part of a system.

\section{[INSERT TABLE 1 ABOUT HERE - if possible on two facing pages, in landscape format]}

\subsubsection{Sender and receiver}

Who are the sender and receiver of communication events? In general, they appear to be spread over the following options (and thus 'values' of the attribute 'type'): human, animal, machine, or part of a system (be this a biological or a computational system or any other kind of system). Some examples are: A communication event in Conversation Analysis requires human senders and receivers, whereas the folk view accepts both human and animal senders and receivers. No discipline only accepts or even requires animals - if animals are considered a possibility, humans are always potential senders and receivers as well. The same applies to machines - disciplines such as NLP and $\mathrm{Al} /$ Information Systems are interested in both human-machine and machine-machine communication, including communication within machines and hence between parts of a computational system. Interestingly, there is only one discipline in our sample where humans are not included: Neuropsychology with the view that sender and receiver form part of a biological system, such as - for the visual system - the sender being visual receptors and the receiver being parts of the brain that are involved in visual processing. Table 1 also includes the option that there is no sender or receiver, in which case an event can be conceptualized as communication without it being essential that there is 
a sender or receiver. None of the disciplines explicitly stated this as an option, but it seems plausible for a few of the disciplines, such as, for example, Al/Information Systems.

Another interesting observation is that the sender and receiver 'type' tables are identical: None of the sampled disciplines puts different restrictions on sender and receiver. However, this is a scenario that can well be imagined - e.g. speech generation would be interested in machine-human communication and machine-generated speech, whereas speech recognition would be looking at human-machine communication and human-generated speech. However, in the range of disciplines as delimited here Speech Science covers both speech generation and speech recognition. This highlights another important aspect in interdisciplinary work and any discussion about disciplinary terminology: The definition of a discipline and its boundaries does have an impact on intradisciplinary terminology, and this should not be underestimated. In the given sample, computational and technical as well as artistic disciplines are conceived rather broadly, whereas, given that the focus of this volume is linguistics, linguistics is split up into a number of subdisciplines. Similar splits can easily be established for the non-linguistic disciplines (cf. above example of speech generation vs. speech recognition), and different evaluations with regards to the dimensions of difference are bound to result.

The other dimension for sender and receiver is summarized as 'criteria'. For the sender, an issue intensely discussed at the workshop and also showing up in this volume (Haugh, this vol.) is intentionality of the sender - whether the sender has to have the intention to send and if so, how this can be verified or known. For all the disciplines except Neuropsychology and those allowing machinemachine communication and communication between parts of a computational system (NLP and $\mathrm{Al} /$ Information Systems), there has to be or generally is an intention to send. This again separates the latter disciplines from the former ones that are focused more on human communication. With regards to how the intention - if present - can be known or verified, the differences in the present disciplines appear to be marginal. For the receiver, the corresponding criterion is whether there has to be an understanding on part of the receiver and if so, how this can be known or verified. Here the difference between knowing or verifying through conscious awareness of the receiver and knowing or verifying through behaviour of the receiver is more prominent. An explanation for this could be that understanding as such is more closely linked to the notion of conscious awareness than to behaviour, whereas in the case of sender intention behavioural verification appears to be as readily accepted as verification through conscious awareness.

\subsubsection{The message}

What is the nature of the message? All disciplines entertain the idea that there is or can be some kind of information involved. However, one can be sure that the notion of 'information' is as diverse as the one of 'communication', and would thus require clarification itself before any closer comparison can take place about what the transmitted 'information' is. Therefore, the nature of the message's information is not discussed further, but we note that all disciplines adopt or potentially adopt the value 'information' for the message attribute 'type'. 
There are, however, a few disciplines where 'information' is not as vital. Speech Pathology, for instance, focuses more on other aspects of the message, such as how the sender performs in the communication process. Other disciplines, although adopting the idea of 'information' being transmitted, are as interested in the incorporation of additional content. One example is Speech Science, where paralinguistic information is often important (e.g. emotions, age, sex, social status, health of the human interactant). Dialog systems might try to pick up what the emotional state of speaker is, and if she gets angry, transfer her to an operator. For yet other disciplines the incorporation of additional aspects is of no interest at all. NLP, as a discipline closely related to Speech Science in many respects, is one of these, and also the 'folk view' would see communication as primarily transmitting information.

The emergence of the message is a very important dimension of difference. This is highlighted by the contributions to this volume, which put it into the centre of discussion: Gould (this vol.), Liddicoat (this vol.), Nevile and Rendle-Short (this vol.), and Haugh (this vol.) all emphasize that the view of a predetermined message being transmitted is incorrect, but that the message is only decipherable in combination with the socio-cultural context or negotiated or emergent in the course of communication. This position stands in sharp contrast to the computational, technological disciplines (NLP, Speech Science, Al/Information Systems) which - potentially in order to stabilize their investigational domain assume a determinate message that is being passed on.

Last but not least another dimension of the message ('character') roughly results in a similar division of disciplines - those that posit that the sent message is the same for the sender and receiver if the communication is successful (e.g., NLP, Speech Science, Introductory Linguistics, folk view) and those that maintain that it is generally not the same for sender and receiver (Intercultural Communication, Pragmatics, Conversation Analysis).

\subsubsection{The underlying system}

Even though the physical nature of the channel (whether it is auditory or visual etc.) seems to be nondecisive for the definition of 'communication' in the different disciplines, the underlying system used for the representation of the message, i.e. the system into which the message is encoded, appears to be of high relevance. For some of the disciplines (in particular, Introductory Linguistics or Gesture/Sign Language) the underlying system even constitutes the focus of study (cf. also Section 3.2).

As most of the represented disciplines study communication including human interactants, the most prevalent type of the underlying system is human language. Only Neuropsychology and Music/Dance do not embrace human language as a possible or required system of communication. Other 'type' values of the underlying system comprise 'other semiotic system (e.g. gesture)' and 'non-linguistic other symbolic system'. In Gesture studies and also in Sign Language research, gestures (and sign 
language signs) are naturally a vital part of the underlying system in question, but also in disciplines such as Speech Science, Intercultural Communication, and Music/Dance, gestures and other semiotic systems are conceivable as being part of the underlying system. Al/Information Systems and Archaeology also look at non-linguistic symbolic systems, such as formal, non-natural languages in the case of $\mathrm{Al} /$ Information Systems and personal and landscape marking in the case of Archaeology. In addition, there is a range of other underlying systems, as for instance (non-symbolic) art expression in Archaeology, the firing of neurons in Neuropsychology, or musical expression and expression via movements in Music/Dance. A classification of the 'other' types of underlying systems would be beneficial, but would require a detailed specification of the systems and of their distinction from symbolic systems, a task which goes beyond the scope of the present paper.

The next obvious point for discussion is how the underlying system came about or was acquired by sender and/or receiver. Three values for this dimension turned out to be relevant: 'biological process', 'exposure; other forms of input (data-driven)' and 'theory, prescriptive rules'. A case of biological process is the emergence of the system in Neuropsychology, although the communicating parts of the neurological system are likely to also necessitate some form of input to form fully. In standard linguistics, there is the generative tradition which sees language acquisition as a biological process, but this view is disputed in other linguistic approaches. Therefore, for Introductory Linguistics and Gesture/Sign Language 'biological process' is marked as a possibility. Intercultural Communication, Conversation Analysis, and Second Language Acquisition are linguistic subdisciplines that are expected to reject the generative view and instead adopt the view that language exposure and language input largely drive language acquisition and hence the emergence of the underlying system. Another example of a clearly data-driven discipline is Speech Science, where the underlying system is learned by the machine through statistical models. The majority of researchers working in NLP also follow a data-driven statistical approach. This again is in contrast to $\mathrm{Al} /$ Information Systems, where theoretical modeling forms the foundation. Prescriptive rules do play a role in Second Language Acquisition, which thus has received plusses for both exposure and theory in Table 1. These are just some examples of the points of view disciplines exhibit with regards to the acquisition or emergence of the underlying system.

Another question is the 'character' of the underlying system. Is it limited or constrained in any way (in contrast to the all-purpose systems human languages are)? Those disciplines that approach communication with the need to formalize - NLP, Speech Science, Al/Information Systems - are working with a constrained underlying system for (at least) machine communication. Also, Second Language Acquisition could be seen as using a limited language and thus underlying system, as language learners are only proficient in a subset of the natural language they acquire. Linguistic subdisciplines such as Introductory Linguistics, Pragmatics, or Conversation Analysis, on the other hand, have a full-fledged natural human language as their object of study and thus as underlying system, i.e. we are not looking at a constrained or limited underlying system (adopting the view that a natural language is an all-purpose system and as such not limited or constrained). 
Finally, the question of the interpretation of the system arises. Are we dealing with a fixed, determinate interpretation of messages encoded with the underlying system, or is the interpretation dynamic, allowing for creativity? Again, disciplines with the need to formalize do work within a more constrained environment and hence with determinate interpretations, as do disciplines such as Speech Pathology that are part of the health care profession. Contrarily, those disciplines that have communication as an object of study - Conversation Analysis, Pragmatics or Intercultural Communication, for example - tend to emphasize the dynamic nature of the interpretation of the underlying system. Haugh (this vol.) is a good example, as he posits that there even is a lack of a priori intentions but that intentions develop through the discourse. And then there are disciplines such as Music/Dance that are founded on the receiver dynamically interpreting the message being transmitted, with dancing seen as a medium for creative communication (Stevens 2005).

\subsection{The study of communication - disciplinary values}

In addition to specifying dimensions of difference about the nature of communication, dimensions related to the study of communication have been taken into account. These - presented in Table 2 are bound to have an impact on how a discipline conceptualizes communication and are thus considered in the following.

\section{[INSERT TABLE 2 ABOUT HERE, in landscape format]}

The discussion of the study of communication is not broken into aspects as the discussion of the nature of communication has been, but, as the table shows, there are four dimensions of difference, referred to as 'focus', 'principal criterion', 'approach', and 'beneficiaries/addressees of research' and their respective values. Naturally, those dimensions are closely connected to and reflect disciplinary values.

The focus of the study looks at the place 'communication' has in the discipline. Communication can be a focus or object of study in itself, or communication and its conceptualization can be a precursor to something else. Disciplines studying communication as an end in itself are, for instance, Intercultural Communication, Pragmatics, Conversation Analysis, and Music/Dance. For those, the problematization of the notion of communication - because focus of the study - is important, whereas for disciplines in which the study of communication is a precursor to some other end it is essential to have a stable conceptualization of communication. Disciplines of the latter kind include the computational, application-driven NLP, Speech Science and Al/Information Systems, but also Neuropsychology and Speech Pathology. Introductory Linguistics, and Gesture/Sign Language for that matter, are also in the latter category, as they are more focused on the underlying system (and its structures) than on communication events themselves.

What is the 'principal criterion' of the study, what is the goal? Is it philosophical rigour, the creation of a formal model, accounting for observations, or practical outcomes (in form of applications, for 
example)? It would be natural to assume that the division of disciplines according to this principal criterion would follow the division of disciplines in the focus dimension - with disciplines seeking philosophical rigour or the creation of a formal model tending to be 'problematizing', and those with practical tasks and goals tending to be 'stabilizing'. However this expectation is not supported by the tables. Although this might hold in the cases of Pragmatics (to some extent), NLP, and Speech Science, Introductory Linguistics is an example of a discipline looking for philosophical rigour and a formal model while seeing communication only as a precursor to other studies rather than a focus in its own right. Intercultural Communication, with communication as a focus of study, is on the other hand oriented towards accounting for observations and targeted at practical outcomes.

The last two dimensions capture whether a discipline's approach is one of formalization or non-formal description, whether qualitative vs. quantitative methods are applied, and who are the beneficiaries or addresses of the research. A formal approach is advocated by the computational disciplines but not by linguistic (sub)disciplines (with the potential exception of Introductory Linguistics and possibly Pragmatics, which do have formal strands). The division is less straightforward in the case of qualitative vs. quantitative methods, as the split goes right through the computational disciplines: NLP and Speech Science mainly use the quantitative approach, whereas Al/Information Systems focuses on the qualitative method - as do all other disciplines under discussion. All disciplines naturally communicate to and have academic peers as beneficiaries, whereas health profession disciplines such as Speech Pathology and classroom oriented disciplines such as SLA also address practitioners, and computational disciplines potential industry partners. The wider community is not so much an explicit target for the communication of results, except through Music/Dance performances.

\section{Discussion}

Perhaps the most important observation to arise from consideration of the tables and their dimensions of difference is their confirmation that views on the nature of communication and how it should be studied do indeed vary widely among the disciplines represented. We have seen above that this variation is not random, suggested reasons for dependencies among the dimensions of difference regarding the nature of communication, and noted some interesting correlations between the Nature of Communication dimensions (Table 1) and the Study of Communication/Disciplinary Values dimensions (Table 2). For example, computational disciplines, i.e. those including machines or parts of computational systems as senders/receivers, tend to take an approach targeted at formalization; while those seeking application in a technical domain tend to assume a more constrained underlying system with a determinate, rather than emergent message.

In one sense, this is hardly surprising. Few would deny that a discipline's overall goals, criteria and metaphysical commitments are likely to influence the way its key theoretical constructs are conceptualized. However it may be worth reflecting a little more on this observation. Moving beyond the obvious implication that there is no single universally correct way to conceptualize communication, it may offer suggestions for successful interdisciplinary collaboration. 
Successful collaboration requires disciplines to move beyond merely tolerating the existence of other points of view, to actively engaging with those other viewpoints. The question is - how to achieve this without losing one's own point of view, compromising one's own disciplinary values, or even threatening one's own identity? This is where we believe the model of intercultural competence may be particularly helpful.

The ability to understand and engage with other cultures without betraying one's own has been characterized as 'finding a third place' (Lo Bianco et al. 1999). This involves creating a metaphorical space in which one is neither entrenched in one's own culture, nor assimilated to another culture, but from which it is possible to observe both cultures with minimal value judgment or stereotyping, and maximal respect. Occupying this third place can engender curiosity about both one's own and the other cultures, and develop understanding of both - without denying the opportunity to return at any time to one's original culture, or indeed to pursue full assimilation to the other. Perhaps the key word here is 'respect', with the appropriate attitude well summarized by one of Holenstein's 'rules of thumb':

One of the first requirements is that one must take members of alien cultures seriously and that one must rather doubt one's own perception than their capacity for logical consistency, goal-orientated rationality and ethical responsibility. (Holenstein 2003)

What does all this mean for the interdisciplinary study of communication? While it may not be possible or even desirable to find a single definition of communication that would satisfy disciplines with differing values, it may well be possible for members of one discipline to temporarily accept the values of another, for the purpose of bringing needed expertise to bear on the other's goal. The keyword here is 'temporarily'. There is no suggestion that members of the 'helping' discipline should abandon their own disciplinary values or projects. Indeed ideally the 'helping' should go both ways, with each discipline able to appreciate the values and goals of the others enough to offer constructive collaboration.

\section{Conclusion}

This paper started from the observation that different disciplines, all considering themselves to be studying communication, may have different ideas about what the term 'communication' actually means. While this is not a problem in itself, it can create a challenge to interdisciplinary collaboration. We have responded to this challenge by creating a set of questions intended to stimulate fruitful interdisciplinary discussion, along the lines of the exchange of ideas at ConCom05, about the various conceptualizations of communication, and by mapping out a typology of these conceptualizations. We hope that this contribution will be useful not only in relation to communication, but also as a model for facilitating mutual understanding about other key terms used by disciplines wishing to collaborate on projects of common interest.

We have further noted that interdisciplinary research on communication is not just about studying communication, but also about doing communication. Human communication in this latter, practical, 
sense has itself been the focus of study by several disciplines (Budd \& Ruben 2003) to which we might turn for inspiration on how to improve the particular kind of communication involved in interdisciplinary collaboration. Noting the analogy between academic disciplines and human cultures, we have suggested that the discipline of applied linguistics, with its wide experience and deep understanding of intercultural communication, might offer a particularly fruitful model for facilitating interdisciplinary communication.

In closing, we note that the entire ConCom05 workshop, including not only prepared lectures but also semi-structured discussion sessions in which some of the ideas elaborated in this paper took form, were recorded and are available online or as a CD. For anyone interested in pursuing an evidencebased study of interdisciplinary communication, these might provide a rich mine of data.

\section{Acknowledgments}

We would like to thank all participants at ConCom05 workshop for their presentations and lively and fruitful discussions. They, the authors in this volume, and two anonymous reviewers have contributed immensely to our understanding of and thinking about interdisciplinary communication and the notion of 'communication' in the different disciplines represented at the workshop and in this volume. It goes without saying that all errors and misinterpretations nevertheless remain our responsibility.

\section{References}

Augsburg T 2006 Becoming Interdisciplinary: An introduction to interdisciplinary studies (second edition) Dubuque, IA: Kendall/Hunt Publishing Company.

Becher T \& PR Trowler 2001 Academic Tribes and Territories: Intellectual enquiry and the culture of disciplines (second edition) Buckingham and Philadelphia, PA: SHRE and Open University Press.

Bennett T, L Grossberg \& M Morris (eds) 2005 New Keywords: A revised vocabulary of culture and society Oxford: Blackwell.

Budd R \& B Ruben (eds) 2003 Interdisciplinary Approaches to Human Communication (second edition) Piscataway, NJ: Transaction Books.

Byram M, A Nichols \& D Stevens (eds) 2001 Developing Intercultural Competence in Practice Clevedon: Multilingual Matters.

Byrne M 1996 What Makes you Say That: Cultural diversity at work (video) SBS.

Chandler D 1994 'The Transmission Model of Communication' http://www.aber.ac.uk/media/Documents/short/trans.htm/ accessed 6 June 2008.

Chandler D 2002 Semiotics: The basics London: Routledge.

Corballis M 2005 Communication on the brain: Lessons from the visual system. Paper presented at ConCom05 (AC Schalley, H Fraser \& D Cogill-Koez 2005).

Curnow $\mathrm{T}$ this vol Communication in introductory linguistics. 
Dale R 2005 Human communication from the perspective of Natural Language Processing. Paper presented at ConCom05 (AC Schalley, H Fraser \& D Cogill-Koez 2005).

Davidson I 2005 Communicating from prehistory: Trust, time, number and distance in sharing meanings. Paper presented at ConCom05 (AC Schalley, H Fraser \& D Cogill-Koez 2005).

Dean R 2005 Becoming-Communication: Some collisions in conceptualising communication in humanities, the arts and cultural studies. Paper presented at ConCom05 (AC Schalley, H Fraser \& D Cogill-Koez 2005).

Derry SJ, CD Schunn \& MA Gernsbacher (eds) 2005 Interdisciplinary Collaboration: An emerging cognitive science Mahwah, $\mathrm{NJ}$ : Lawrence Erlbaum Associates.

Eisenchlas $\mathrm{S}$ this vol Conceptualising 'communication' in Second Language Acquisition.

Eklund P 2005 Conceptualisation of communication in computation: A case study in Formal Concept Analysis. Paper presented at ConCom05 (AC Schalley, H Fraser \& D Cogill-Koez 2005).

FitzGerald H 2003 How Different are We: Spoken discourse in intercultural communication Cleveland: Multilingual Matters.

Goddard C this vol The 'communication concept' and the 'language concept' in everday English.

Gould $\mathrm{J}$ this vol There is more to communication than tongue placement and 'Show and Tell': Discussing communication from a speech pathology perspective.

Haugh M this vol Intention(ality) and the conceptualisation of communication in pragmatics.

Holenstein E 2003 'A Dozen Rules of Thumb for Avoiding Intercultural Misunderstandings' Polylog: Forum for Intercultural Philosophy 4.

Klein JT 1990 Interdisciplinarity: History, theory and practice Detroit: Wayne State University Press.

Lattuca LR 2001 Creating Interdisciplinarity: Interdisciplinary research and teaching among college and university faculty Nashville, TN: Vanderbilt University Press.

Lele S \& RB Norgaard 2005 'Practicing interdisciplinarity' Bioscience 55(11): 967-975.

Liddicoat AJ this vol Communication as culturally contexted practice: A view from intercultural communication.

Lo Bianco J, C Crozet \& A Liddicoat (eds) 1999 Striving for the third place: Intercultural competence through language education Canberra: Language Australia.

Lovell L 2005 Letting our hands do the talking: Communication through gesture. Paper presented at ConCom05 (AC Schalley, H Fraser \& D Cogill-Koez 2005).

Marris P \& S Thornham 1996 Media Studies: A reader Edinburgh: Edinburgh University Press.

Miller G 2003 'The cognitive revolution: a historical perspective' Trends in Cognitive Sciences 7(3): 141-144.

Moran J 2001 Interdisciplinarity: The new critical idiom London: Routledge.

Nevile $M$ \& $J$ Rendle-Short this vol A conversation analysis view of communication as jointly accomplished social interaction: An unsuccessful proposal for a social visit.

Rogers L \& G Kaplan 1998 Not Only Roars and Rituals: Communication in animals Sydney: St Leonards.

Schalley AC, H Fraser \& D Cogill-Koez 2005 Conceptualising Communication: Building Crossdisciplinary Understanding in Human Communication Science (ConCom05). An 
interdisciplinary workshop sponsored by the Human Communication Science Network and the

UNE Language and Cognition Research Centre (http://www.hcsnet.edu.au/workshops/ConCom05) University of New England Armidale.

Schembri A \& T Johnston 2005 Language and communication in signed language linguistics. Paper presented at ConCom05 (AC Schalley, H Fraser \& D Cogill-Koez 2005).

Shannon CE \& W Weaver 1949 A Mathematical Model of Communication Urbana, IL: University of Illinois Press.

Stevens K 2005 Visible thought: Communication and the performing arts: An experimental psychologists' view of contemporary dance. Paper presented at ConCom05 (AC Schalley, H Fraser \& D Cogill-Koez 2005).

Todd Z, B Nerlich, S McKeown \& DD Clarke 2001 Mixing Methods in Psychology: The integration of qualitative and quantitative methods in psychology Hove and New York: Psychology Press.

Wagner, M 2005 Spoken language technology, speech science and human communication. Paper presented at ConCom05 (AC Schalley, H Fraser \& D Cogill-Koez 2005).

Weingart P \& N Stehr 2000 Practising Interdisciplinarity Toronto: University of Toronto Press.

Wierzbicka A 1997 Understanding Cultures Through Their Key Words: English, Russian, Polish, German, Japanese New York: Oxford University Press. 
Table 1. Nature of Communication

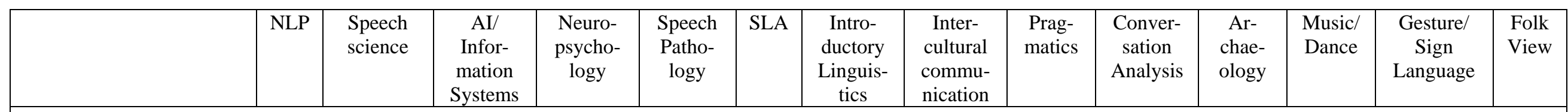

\section{What is the nature of the sender?}

\begin{tabular}{|l|c|}
\hline \multicolumn{2}{|l|}{ Type } \\
\hline none & - \\
\hline human & + \\
\hline animal & - \\
\hline machine & + \\
\hline part of a system (biologic., & +
\end{tabular}

part of a system (biologic.,

computational, etc.)

Criteria for sending

intention (known / verified

through conscious

awareness by sender)

intention (known / verified

through sender behaviour)

no intention to send

\begin{tabular}{|c|c|c|c|c|c|c}
- & - & $?$ & $?$ & - & - & \\
\hline+ & + & + & - & + & + & \\
\hline- & - & $?$ & - & - & - & \\
+ & + & + & - & - & - & \\
+ & $?$ & + & + & - & - & + \\
& & & & & & \\
\hline
\end{tabular}

\begin{tabular}{|c|c|c|c|c|c|c|c|}
\hline & + & + & $?$ & - & + & + & + \\
\hline r) & + & + & + & $?$ & + & + & + \\
\hline & $?$ & - & $?$ & + & - & - & - \\
\hline
\end{tabular}

\begin{tabular}{|c|c|c|c|c|c|c|}
\hline- & - & - & $?$ & $?$ & $?$ & - \\
\hline+ & + & + & + & + & + & + \\
\hline- & - & - & - & - & $?$ & + \\
\hline- & - & - & - & $?$ & - & - \\
\hline- & - & - & - & - & - & - \\
\hline
\end{tabular}

What is the nature of the receiver?

Type [the same classification as for the sender resulted - refer to the respective rows in the 'nature of sender' section]

Criteria for receiving

\begin{tabular}{|c|c|c|c|c|c|c|c|c|c|c|c|c|c|c|}
\hline $\begin{array}{l}\text { understanding (known / } \\
\text { verified through conscious } \\
\text { awareness by receiver) }\end{array}$ & + & + & ? & ? & + & + & + & + & + & + & ? & + & + & + \\
\hline $\begin{array}{l}\text { understanding (known / } \\
\text { verified through receiver } \\
\text { behaviour }\end{array}$ & + & - & + & $?$ & $?$ & - & - & $?$ & $?$ & $?$ & $?$ & + & + & + \\
\hline none & $?$ & - & $?$ & + & - & - & $?$ & - & - & - & + & - & - & - \\
\hline
\end{tabular}

${ }^{1}$ human as information system 


\section{What is the content of the message?}

Type

\begin{tabular}{|c|c|c|c|c|c|c|c|c|c|c|c|c|c|c|}
\hline 'information’ & + & + & + & + & $?$ & + & + & $?$ & + & + & + & $?$ & + & + \\
\hline additional aspects & - & + & - & $?$ & + & $?$ & $?$ & + & - & + & + & + & - & - \\
\hline
\end{tabular}

Emergence of message content

determinate (e.g. by

sender, parts of message)

negotiated in / emergent

from exchange

only decipherable in

combination with context

Character

unchanged throughout

exchange (same for sender

and receiver)

\begin{tabular}{|c|c|c|c|c|c}
+ & + & + & + & $?$ & + \\
\hline- & - & - & - & - & $?$ \\
\hline- & - & - & $?$ & + & + \\
\hline
\end{tabular}

\begin{tabular}{l|c|c|}
+ & + & \\
\hline$?$ & - & \\
\hline+ & - &
\end{tabular}

\begin{tabular}{c|c|c|c|}
- & $?$ & - & \\
\hline- & + & + & \\
\hline+ & + & + & \\
\hline
\end{tabular}

\begin{tabular}{|c|c|c|c|}
+ & \\
\hline- & & \\
\hline+ & &
\end{tabular}

\begin{tabular}{|c|c|c|}
\hline- & + & + \\
\hline+ & + & - \\
\hline+ & - & - \\
\hline
\end{tabular}

What is the nature of the underlying system? ${ }^{2}$

\section{Type}

\begin{tabular}{|c|c|c|c|c|c|c|c|c|c|c|c|c|c|c|}
\hline human language & + & + & ? & - & + & + & + & + & + & + & + & - & + & + \\
\hline $\begin{array}{l}\text { other semiotic system (e.g. } \\
\text { gesture) }\end{array}$ & - & $?$ & - & - & $?$ & - & - & $?$ & - & - & - & $?$ & + & $?$ \\
\hline non-ling. symbolic system & + & ? & + & - & - & - & - & - & - & - & + & ? & - & - \\
\hline other & - & - & - & + & - & - & - & - & - & + & + & + & - & - \\
\hline
\end{tabular}

other

\begin{tabular}{cccc}
- & - & - & - \\
\hline
\end{tabular}

\begin{tabular}{|c|c|c|c|c|c|c|c|c|c|c|c|c|c|c|}
\hline biological process & - & $?$ & - & + & + & - & $?$ & - & - & - & + & + & $?$ & - \\
\hline $\begin{array}{l}\text { exposure; other forms of } \\
\text { input (data-driven) }\end{array}$ & + & + & $?$ & $?$ & + & + & + & + & + & + & + & + & + & + \\
\hline theory; prescriptive rules & + & ? & + & - & - & + & - & $?$ & $?$ & $?$ & $?$ & + & $?$ & + \\
\hline \multicolumn{15}{|l|}{ Character } \\
\hline limited or constrained & + & + & + & + & - & + & - & - & - & - & + & + & - & $?$ \\
\hline determinate interpretation & + & + & + & $?$ & + & + & + & $?$ & $?$ & $?$ & + & - & + & + \\
\hline dynamic interpretation & - & - & - & $?$ & $?$ & - & - & + & + & + & - & + & - & - \\
\hline
\end{tabular}

${ }^{2}$ Although one may distinguish the channel for transferring the message from the underlying system in which the message is transferred, we disregard the channel and its type (visual, auditory, etc.) in our dimensions of difference, as it can generally vary without changing the definition or understanding of the nature of communication. 
Table 2. Study of communication / Disciplinary values

\begin{tabular}{|c|c|c|c|c|c|c|c|c|c|c|c|c|c|}
\hline NLP & $\begin{array}{l}\text { Speech } \\
\text { science }\end{array}$ & $\begin{array}{c}\text { AI/ } \\
\text { Infor- } \\
\text { mation } \\
\text { Systems }\end{array}$ & $\begin{array}{c}\text { Neuro- } \\
\text { psycho- } \\
\text { logy }\end{array}$ & $\begin{array}{c}\text { Speech } \\
\text { Patho- } \\
\text { logy }\end{array}$ & SLA & $\begin{array}{l}\text { Intro- } \\
\text { ductory } \\
\text { Linguis- } \\
\text { tics }\end{array}$ & $\begin{array}{l}\text { Inter- } \\
\text { cultural } \\
\text { commu- } \\
\text { nication }\end{array}$ & $\begin{array}{l}\text { Prag- } \\
\text { matics }\end{array}$ & $\begin{array}{l}\text { Conver- } \\
\text { sation } \\
\text { Analysis }\end{array}$ & $\begin{array}{l}\text { Ar- } \\
\text { chae- } \\
\text { ology }\end{array}$ & $\begin{array}{l}\text { Music/ } \\
\text { Dance }\end{array}$ & $\begin{array}{c}\text { Gesture/ } \\
\text { Sign } \\
\text { Language }\end{array}$ & $\begin{array}{c}\text { Folk } \\
\text { View } \\
3\end{array}$ \\
\hline
\end{tabular}

Focus

\begin{tabular}{|c|c|c|c|c|c|c|c|c|c|c|c|c|c|}
\hline $\begin{array}{l}\text { end in itself } \\
\text { precursor to something }\end{array}$ & - & - & - & - & ? & + & - & + & + & + & + & + & - \\
\hline $\begin{array}{l}\text { precursor to something } \\
\text { else }\end{array}$ & + & + & + & + & + & + & + & $?$ & $?$ & $?$ & - & - & + \\
\hline
\end{tabular}

else

Principal criterion

\begin{tabular}{|c|c|c|c|c|c|c|c|c|c|c|c|c|c|}
\hline \multirow{2}{*}{$\begin{array}{l}\text { philosophical rigour } \\
\text { a formal model }\end{array}$} & - & - & ? & - & - & - & + & - & + & - & ? & - & + \\
\hline & ? & ? & + & ? & ? & ? & + & ? & + & - & ? & - & + \\
\hline $\begin{array}{l}\text { accounting for } \\
\text { observations }\end{array}$ & $?$ & $?$ & - & + & + & - & + & + & + & + & + & $?$ & + \\
\hline $\begin{array}{l}\text { practical outcomes or } \\
\text { applications }\end{array}$ & + & + & + & - & + & + & - & + & - & + & - & + & - \\
\hline
\end{tabular}

Approach

formalisation

non-formal description

qualitative method

quantitative method

\begin{tabular}{|c|c|c|c|c|c|c|c|}
\hline & + & + & + & $?$ & - & - & + \\
\hline- & - & - & $?$ & + & + & + \\
\hline$?$ & $?$ & + & + & + & + & + \\
\hline+ & + & $?$ & - & - & - & - \\
\hline
\end{tabular}

\begin{tabular}{|c|c|}
- & \\
+ & \\
+ & \\
- &
\end{tabular}

Beneficiaries/addressees of research

\begin{tabular}{|c|c|c|c|c|c|c|c|c|c|c|c|c|c|}
\hline \multirow{2}{*}{$\begin{array}{l}\text { academic peers } \\
\text { industry partners }\end{array}$} & + & + & + & + & + & + & + & + & + & + & + & + & + \\
\hline & + & + & + & - & $?$ & - & - & - & - & - & - & + & - \\
\hline practitioners & - & - & - & - & + & + & - & + & - & - & - & + & - \\
\hline wider community & - & - & - & - & - & - & - & $?$ & - & - & $?$ & + & - \\
\hline
\end{tabular}

\footnotetext{
${ }^{3}$ 'Folk view' is greyed out for the study of communication, as no explicit study of communication is undertaken.
} 